EPJ Web of Conferences 13, 07002 (2011)

DOI: $10.1051 /$ epjconf/20111307002

(c) Owned by the authors, published by EDP Sciences, 2011

\title{
Deflected Jets or Hot Spots? Conical Correlations of Hard Trigger Particles
}

\author{
Barbara Betz ${ }^{\mathrm{a}}$ \\ Department of Physics, Columbia University, New York, 10027, USA
}

\begin{abstract}
The double-peak structure observed in soft-hard dihadron correlations was recently studied intensively in order to learn more about the jet-induced medium excitation in ultrarelativistic heavy-ion collisions. Experimental data shows that the double-peak structure obtained for soft trigger particles coalesces into one peak for harder trigger particles. We demonstrate that this effect occurs when averaging over many jet events in a transversally expanding background, while a hot spot scenario always leads to two distinct peaks. This suggests to study soft-hard correlations induced by heavy-flavor jets with those generated by light-flavor jets at RHIC and LHC in order to really disentangle medium effects from jets.
\end{abstract}

The hot and dense medium created in ultrarelativistic heavy-ion collisions [1-6], which is most likely the quarkgluon plasma (QGP), can be probed with the help of jets. It is assumed that those jets are created in the early stages of the collisions and interact with the expanding system. At RHIC it was found that the medium behaves as a nearly perfect fluid [9] and that it is opaque to jets [7], like at the LHC [8]. This raises the possibility of studying medium properties using the correlations of soft and hard particles.

The interest in the experimental multi-particle correlations [10-13] is based on the double-peak structure found at angles opposite to the trigger jet, which has been suggested as a signal for the creation of Mach cones [14,15].

In a fluid with low viscosity, Mach cones are generated by the interference of sound waves resulting from the energy deposited by a supersonic jet. They should lead to an excess of low- $p_{T}$ hadrons which are emitted at an angle $\pi-\phi_{M}$ with respect to the trigger jet. The Mach-cone angle $\phi_{M}$ is given by Mach's law, $\cos \phi_{M}=c_{s} / v_{\text {jet }}$, providing a possibility to extract the speed of sound $c_{s}$.

Experimental multi-particle correlations were studied intensively. It was shown that the position of the away-side peaks does not change with $p_{T}^{a s s o c}$ (excluding Cherenkov gluon radiation as a source for the double-peak structure), but strongly depends on $p_{T}^{\text {trig }}$. While a clear double-peak structure is seen for smaller $p_{T}^{\text {trig }}\left(3<p_{T}^{\text {trig }}<4 \mathrm{GeV}\right)$, this structure coalesces into one peak for larger $p_{T}^{\text {trig }}(6<$ $\left.p_{T}^{\text {trig }}<10 \mathrm{GeV}\right)[16,17]$.

Recently, however, it has been shown [18] that the experimentally observed two-peak structure for small $p_{T}^{\text {trig }}$ and $p_{T}^{a s s o c}$ can also be obtained in two-particle correlations without considering jets, but hot spots which occur due to the fluctuation of initial conditions. The flow created by the hot spot will interfere with the flow of the expanding

\footnotetext{
a e-mail: betz@phys.columbia.edu
}

medium, forming a conical structure and resulting in two peaks on the away-side.

Moreover, it was suggested in Ref. [19] that the triangular flow $\left(v_{3}\right)$ could also lead to the away-side features observed in experimental data. This effect was studied in detail, both experimentally [20] and theoretically [21,22]. Unfortunately, it seems to yet remain inconclusive if the conical structure will still be present after the subtraction of the triangular flow. This question can only be resolved after an experimentally extracted $v_{3}$ component is subtracted from the measured data.

In the following, however, we will demonstrate an effect that might lead, for very central events, to a weakening of the double-peak structure at larger $p_{T}^{\text {trig }}$, considering jets traversing through the medium. It will clearly differ from a hot spot event (as presented below) and is closely connected to the path length dependence of a jet $[23,24]$.

Previous calculations [25-34] have shown that the formation of a conical structure on the away-side of softhard correlations can be very sensitive to the underlying assumptions about the jet-medium interaction [35]. While in case of a static medium a diffusion wake moving in the opposite trigger-jet direction may overwhelm any signal from the Mach cone leading to a single peak on the awayside [25,31-34], the strong longitudinal and transverse expansion of the QGP can distort the Mach-cone signal [27, $36,37]$. This diffusion wake is universal to strongly and weakly-coupled energy loss [31].

Assuming that the energy lost by the jet thermalizes quickly [10], we solve the conservation equations

$$
\partial_{\mu} T^{\mu v}=S^{v}
$$

of the energy-momentum tensor $T^{\mu v}=(e+p) u^{\mu} u^{v}-p g^{\mu v}$, where $u^{\mu}$ is the four-velocity of the fluid, using the $(3+1)$ dimensional hydrodynamic SHASTA algorithm [38] for an ideal gas $\operatorname{EoS}(p=e / 3)$ of massless $S U(3)$ gluons. $S^{v}$ denotes the energy and momentum deposited by a jet. We 
choose the following ansatz

$$
S^{v}(x)=\int_{\tau_{i}}^{\tau_{f}} d \tau \frac{d M^{v}}{d \tau} \frac{u_{\alpha} j^{\alpha}}{u_{0, \beta} j_{0}^{\beta}} \delta^{(4)}\left[x-x_{\mathrm{jet}}(\tau)\right],
$$

with the proper-time interval of the jet evolution $\tau_{f}-\tau_{i}$, the (constant) energy and momentum loss rate $d M^{v} / d \tau=$ $(d E / d \tau, d \mathbf{M} / d \tau)$, and the location of the jet $x_{\text {jet }}$. Here, $j^{\alpha}$ is the four-current of color charges and $u_{0}^{\beta}, j_{0}^{\beta}$ are the initial four-velocity and four-current of color charges at the center of the system, respectively. Thus, the factor $u_{\alpha} j^{\alpha} /\left(u_{0, \beta} j_{0}^{\beta}\right)$ takes into account that the medium expands and cools, reducing the energy-momentum loss rate. In non-covariant notation, Eq. (2) reads

$$
\begin{aligned}
S^{v}(t, \mathbf{x}) & =\frac{1}{(\sqrt{2 \pi} \sigma)^{3}} \\
& \exp \left\{-\frac{\left[\mathbf{x}-\mathbf{x}_{\mathrm{jet}}(t)\right]^{2}}{2 \sigma^{2}}\right\}\left(\frac{d E}{d t}, \frac{d M}{d t}, 0,0\right)\left[\frac{T(t, \mathbf{x})}{T_{\max }}\right]^{3} .
\end{aligned}
$$

In the following, $\sigma=0.3 \mathrm{fm}$.

We investigate an expanding medium with an initial transverse energy density profile given by the Glauber model for a maximum temperature of either $T=200 \mathrm{MeV}$ $(\mathrm{Au}+\mathrm{Au})$ or $T=176 \mathrm{MeV}(\mathrm{Cu}+\mathrm{Cu})$. Note that the exact value of the initial temperature does not play an important role for the analysis since we are considering an ideal gas EoS. In the longitudinal direction, the system is assumed to be a cylinder, elongated over the whole grid. With this assumption, we minimize the effect of longitudinal flow. A temperature cut of $T_{\text {cut }}=130 \mathrm{MeV}$ is applied to ensure that no energy-momentum deposition takes place outside the medium. Since it was shown in Ref. [34] that jet deceleration does not lead to significant changes in the particle correlations after freezeout, we consider that the jets move at a constant velocity through the expanding medium.

However, we assume that each parton moving through the QGP will eventually be completely thermalized after the deposition of all its initial energy. This is an important difference to the ansatz chosen in Ref. [26] where the jet was energetic enough to punch through the medium.

Here we consider the jet to be generated by a $5 \mathrm{GeV}$ or $11.4 \mathrm{GeV}$ parton which corresponds to a trigger- $p_{T}$ of $3.5 \mathrm{GeV}$ and $8 \mathrm{GeV}$, respectively, assuming that, after fragmentation, the leading hadron carries $\sim 70 \%$ of the parton's energy.

Since experiment can trigger on the jet direction, but not on the location where the jet was formed, one has to consider different jet trajectories pointing along the same direction but originating from different points in the transverse plane [39]. We parametrize these trajectories as

$$
x=r \cos \phi \quad y=r \sin \phi,
$$

where $r=5 \mathrm{fm}$ is chosen to account for surface bias and consider different values for the azimuthal jet angle with respect to the trigger axis (which is chosen to be the negative $x$-axis). Here we denote all jets travelling at $\phi=$
$90, \ldots, 165$ degrees (with a $\Delta \phi=15$ degrees) as jets propagating through the upper half of the medium and jets between $\phi=195, \ldots, 270$ degrees as those going through the lower half of the medium.

After the hydrodynamic evolution, the fluid is converted into particles using the Cooper-Frye (CF) prescription [40] at a constant time (isochronous freeze-out) which leads to the single-inclusive particle spectrum $d N /\left(p_{T} d p_{T} d y d \phi\right)$.

One major difference between the experimental situation and the hydrodynamical calculation proposed above is that the trajectory of the jet is not known in the first case. Thus, one has to measure the azimuthal correlation between hard particles produced by the trigger jet and soft particles produced by the associated jet. We mimic the hardsoft correlation function by convoluting the single-inclusive particle spectrum (which only considers the away-side particles) with a function representing the near-side jet,

$$
f(\phi)=\frac{1}{\sqrt{2 \pi \Delta \phi^{2}}} \exp \left(-\frac{\phi^{2}}{2 \Delta \phi^{2}}\right),
$$

$(\Delta \phi=0.4)$, resulting in a two-particle correlation function

$$
C_{2}(\phi)=A f(\phi)+\int_{0}^{2 \pi} d \phi^{\star} \frac{d N}{p_{T} d p_{T} d y d\left(\phi-\phi^{\star}\right)} f\left(\phi^{\star}\right),(
$$

where $(A, \Delta \phi)$ are chosen to simulate the near-side correlation. This function is then event-averaged (indicated by $\langle\cdot\rangle)$, background-subtracted, and normalized, leading to the averaged two-particle correlation function

$$
\langle C F(\phi)\rangle=\mathcal{N}\left[\left\langle C_{2}(\phi)\right\rangle-\frac{d N_{\text {back }}}{p_{T} d p_{T} d y d \phi}\right],
$$

where $d N_{\text {back }} /\left(p_{T} d p_{T} d y d \phi\right)$ is the single-inclusive particle spectrum for an event without jets and inverse of $\mathcal{N}$ is $\mathcal{N}^{-1}=d N_{\text {back }} /\left(p_{T} d p_{T} d y\right)$.

Figure 1 shows the two-particle correlation function for a $p_{T}^{\text {trig }}=3.5 \mathrm{GeV}$ (left) and a $p_{T}^{\text {trig }}=8.0 \mathrm{GeV}$ (right) assuming a $p_{T}^{a s s o c}=2 \mathrm{GeV}$. The jets are considered to propagate with $v=0.999$, depositing energy and momentum into the medium according to Eq. (3) with $d E / d t=$ $1 \mathrm{GeV} / \mathrm{fm}$ and $d M / d t=1 / v d E / d t$. In both cases we observe a double-peak structure resembling a Mach-cone signal, but the peak-to-valley ratio is much smaller for the larger $p_{T}^{\text {trig }}$. The cone-like signal is a consequence of the different contributions of the jet trajectories that are shown in the lower panels of Fig. 1. Those jets traversing the upper half of the medium add up to a peak at an angle smaller than 180 degrees (long-dashed blue lines in the upper panels of Fig. 1), while the contributions from the jets traversing the lower half of the medium (short-dashed magenta lines in the upper panels of Fig. 1) lead to a peak at an angle larger than 180 degrees. The gap between those two peaks depends on how much the transversally expanding medium deflects the matter in the disturbances caused by the jet as well as on $p_{T}^{a s s o c}$.

For a larger $p_{T}^{\text {trig }}$ the jet traversing the middle of the medium (red line in the lower right panel of Fig. 1) may 

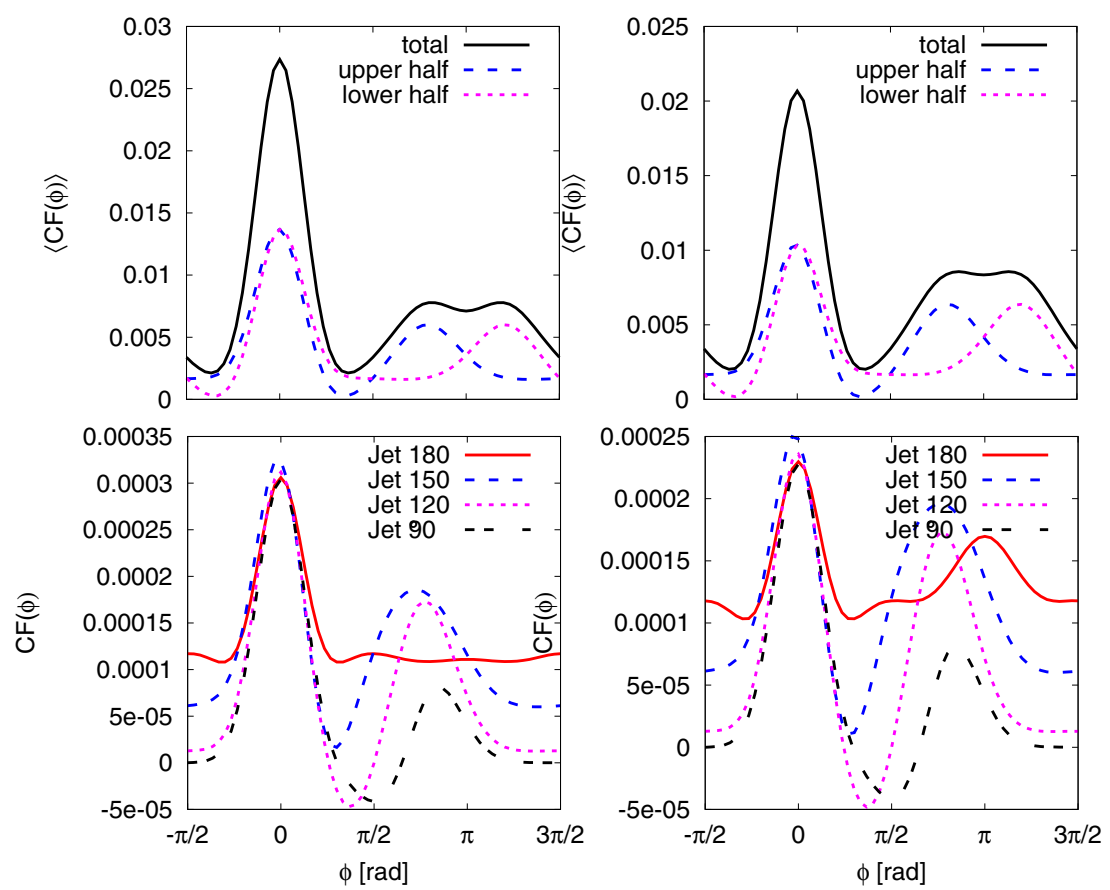

Fig. 1. The two-particle correlation function (solid black line) for a $p_{T}^{\text {trig }}=3.5 \mathrm{GeV}$ (left panel) [37] and for a $p_{T}^{\text {trig }}=8 \mathrm{GeV}$ (right panel), assuming that the associated particle is $p_{T}^{a s s o c}=2 \mathrm{GeV}$. The long-dashed blue and short-dashed magenta lines in the upper panels represent the averaged contribution from jets traversing only the upper or the lower half of the medium, respectively. The unaveraged two-particle correlation function is shown in the lower panels from four representatively chosen different jet trajectories in the upper half of the medium.

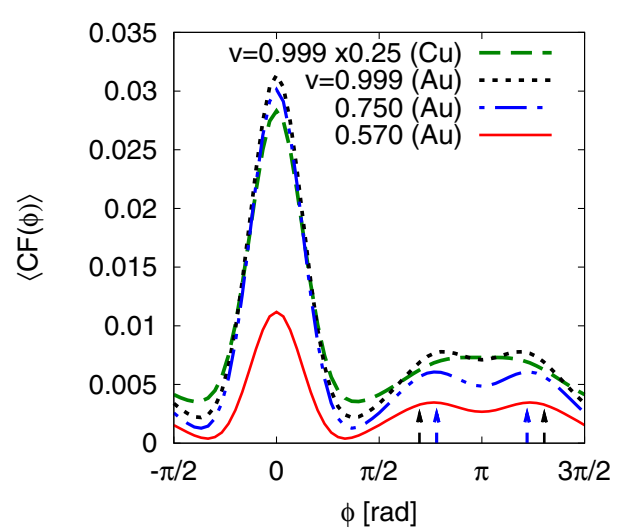

Fig. 2. The two-particle correlation function, Eq. (7), for light jets travelling at $v=0.999$ in central $\mathrm{Cu}+\mathrm{Cu}$ collisions (longdashed green line) as well as central $\mathrm{Au}+\mathrm{Au}$ collisions (shortdashed black line), and $b$-jet propagating at $v=0.75$ (dash-dotted blue line) and $v=0.57$ (solid red line) for a $p_{T}^{\text {assoc }}=2 \mathrm{GeV}$. For the supersonic jets, the arrows indicate the emission angles obtained by Mach's law. In case of the $\mathrm{Cu}+\mathrm{Cu}$, the double-peak structure only appears for larger $p_{T}^{a s s o c}$ due to thermal smearing [37].

reach that part of the medium where the background flow of the expanding system is parallel to the flow created by the diffusion wake $[37,41]$, enhancing its impact and causing a contribution opposite to the trigger jet which fills up the double-peak structure. This effect might be seen in the data $[16,17]$, leading to a two-peak structure for small $p_{T}^{\text {trig }}$ and just one broad away-side peak for large $p_{T}^{\text {trig }}$.

Thus, the conical shape results from the averaging over many different jet events in an expanding medium [37]. It even appears for subsonic jets (see Fig. 2) which demonstrates that the effect cannot be due to a true Mach cone or used to conclusively distinguish between different jet deposition scenarios [37].

As discussed above, the two peak structure on the away side of azimuthal correlations for small $p_{T}^{\text {trig }}$ and $p_{T}^{\text {assoc }}$ can also be obtained due to the evolution of a hot spot [18]. However, the relevant question is if such a double-peak structure also coalesces into one peak for larger $p_{T}^{\text {trig }}$.

To check this, we basically replaced a jet in the above setup of most central collisions by a hot spot, choosing different $\Delta e / e_{0}$. Please note that the actual position of the hot spot is irrelevant in this case and it is also not necessary to average over many events since there is no trigger jet axis and thus all events can be converted into each other due to rotational symmetry.

Fig. 3 shows the two-particle correlation function for such a hot spot event (blue dashed lines) assuming a $p_{T}^{\text {assoc }}=$ $2.0 \mathrm{GeV}$ and a $p_{T}^{\text {trig }}=3.5 \mathrm{GeV}$ (left panel) as well as a $p_{T}^{\text {trig }}=8.0 \mathrm{GeV}$ (right panel), obtained according to

$$
\frac{1}{N} \frac{d N}{d \Delta \phi}=\frac{1}{N} \int \frac{d N}{d \phi} \frac{d N}{d(\Delta \phi-\phi)} d \phi
$$



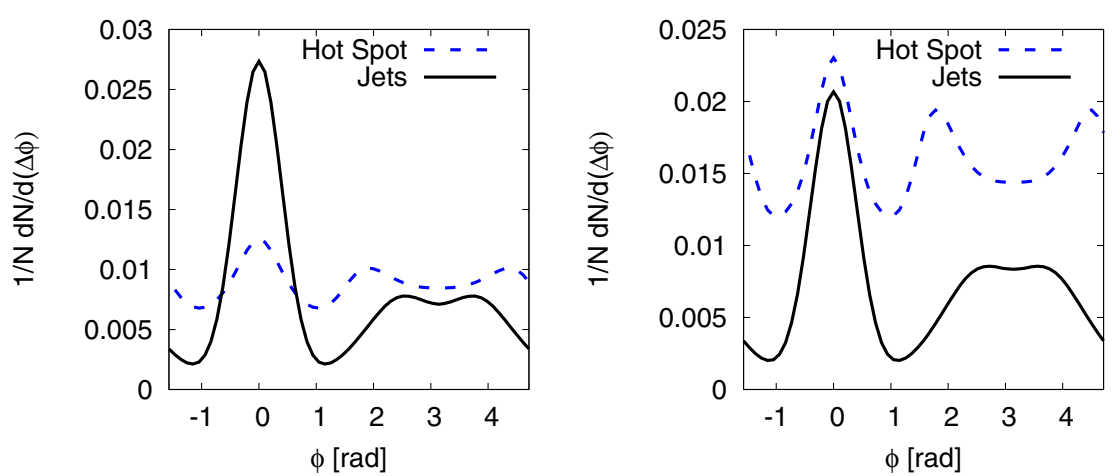

Fig. 3. Two-particle correlation function from a hot spot event (dashed blue lines) and averaged jet events (black solid lines), assuming a $p_{T}^{\text {assoc }}=2.0 \mathrm{GeV}$ and $p_{T}^{\text {trig }}=3.5 \mathrm{GeV}$ (left panel) as well as $p_{T}^{\text {trig }}=8.0 \mathrm{GeV}$ (right panel).

and compared with the averaged jet events from Fig. 1 (black solid line). Here we chose $\Delta e / e_{0}=6$, other ratios give similar results.

As can be seen, the double-peak structure is more pronounced in case of a hot spot and gets even stronger for larger $p_{T}^{\text {trig }}$, in contrast to the averaged jet events where the two peak structure starts to coalesce into one peak for larger $p_{T}^{\text {trig }}$ like seen in the data. The position of the two peaks in the hot-spot scenario is due to the relative flow between the hot spot and the background. Therefore, it stays nearly constant for different $p_{T}^{\text {trig }}$.

However, it seems rather unlikely that a hot spot creates particles with very large $p_{T}$. But for small $p_{T}^{\text {trig }}[16,17]$ a superposition of fluctuating events (hot spots) and jets might probably lead to a rather clean double-peak structure. This should also be seen at the LHC.

It is important to note in this context that the effect of triangular flow and hot spots are very closely linked to each other and might actually not be disentangled. Each hot spot (and thus fluctuating initial conditions) will lead to a nonzero triangular flow.

In conclusion, we have shown that a double-peak structure on the away side of soft-hard correlations obtained via averaging over different jet events in which the particles are emitted from the deflected wakes created by jets [37] coalesces into one peak for larger $p_{T}^{\text {trig }}$ as seen in experimental data $[16,17]$, in contrast to the the two-peak structure obtained from hot spots. Since such a distinction is not possible experimentally, it is necessary to study soft-hard correlations induced by heavy-flavor tagged jets [42] with those induced by light-flavor jets at RHIC and LHC in order to disentangle the medium effects (hot spots) from jets and to test if a conical correlation occurs even for subsonic "jets" [37].

B.B. thanks J. Noronha, G. Torrieri, M. Gyulassy, and D. Rischke for all the discussions and acknowledges support from the Alexander von Humboldt foundation via a Feodor Lynen fellowship and DOE under Grant No. DEFG02-93ER40764.

\section{References}

1. I. Arsene et al. [BRAHMS Collaboration], Nucl. Phys. A 757, 1 (2005).

2. K. Adcox et al. [PHENIX Collaboration], Nucl. Phys. A 757, 184 (2005).

3. B. B. Back et al., Nucl. Phys. A 757, 28 (2005).

4. J. Adams et al. [STAR Collaboration], Nucl. Phys. A 757, 102 (2005).

5. K. Aamodt et al. [The ALICE Collaboration], arXiv:1011.3916 [nucl-ex].

6. K. Aamodt et al. [The ALICE Collaboration], arXiv:1011.3914 [nucl-ex].

7. M. Gyulassy and L. McLerran, Nucl. Phys. A 750, 30 (2005); E. V. Shuryak, Nucl. Phys. A 750, 64 (2005).

8. K. Aamodt et al. [ALICE Collaboration], arXiv:1012.1004 [nucl-ex].

9. P. Romatschke and U. Romatschke, Phys. Rev. Lett. 99, 172301 (2007).

10. J. Adams et al. [STAR Collaboration], Phys. Rev. Lett. 95, 152301 (2005).

11. S. S. Adler et al. [PHENIX Collaboration], Phys. Rev. Lett. 97, 052301 (2006).

12. A. Adare et al. [PHENIX Collaboration], Phys. Rev. C 78, 014901 (2008).

13. B. I. Abelev et al. [STAR Collaboration], Phys. Rev. Lett. 102, 052302 (2009).

14. H. Stoecker, Nucl. Phys. A 750, 121 (2005).

15. J. Casalderrey-Solana, E. V. Shuryak and D. Teaney, J. Phys. Conf. Ser. 27, 22 (2005) [Nucl. Phys. A 774, 577 (2006)].

16. A. Adare et al. [PHENIX Collaboration], Phys. Rev. C 78, 014901 (2008).

17. M. M. Aggarwal et al. [STAR Collaboration], Phys. Rev. C 82, 024912 (2010).

18. J. Takahashi et al., Phys. Rev. Lett. 103, 242301 (2009).

19. B. Alver and G. Roland, Phys. Rev. C 81, 054905 (2010) [Erratum-ibid. C 82, 039903 (2010)].

20. H. Agakishiev et al., arXiv:1010.0690 [nucl-ex].

21. J. Xu and C. M. Ko, arXiv:1011.3750 [nucl-th].

22. G. L. Ma and X. N. Wang, arXiv:1011.5249 [nucl-th].

23. A. Sickles, Eur. Phys. J. C 61, 583 (2009). 
24. S. Afanasiev et al. [PHENIX Collaboration], Phys. Rev. Lett. 99, 052301 (2007).

25. J. Casalderrey-Solana, E. V. Shuryak and D. Teaney, arXiv:hep-ph/0602183.

26. A. K. Chaudhuri and U. Heinz, Phys. Rev. Lett. 97, 062301 (2006).

27. T. Renk and J. Ruppert, Phys. Lett. B 646, 19 (2007); Phys. Rev. C 76, 014908 (2007).

28. R. B. Neufeld, B. Muller and J. Ruppert, Phys. Rev. C 78, 041901 (2008).

29. S. S. Gubser, S. S. Pufu and A. Yarom, Phys. Rev. Lett. 100, 012301 (2008).

30. J. Noronha, M. Gyulassy and G. Torrieri, Phys. Rev. Lett. 102, 102301 (2009).

31. B. Betz, M. Gyulassy, J. Noronha and G. Torrieri, Phys. Lett. B 675, 340 (2009).

32. D. Molnar, AIP Conf. Proc. 1182, 791 (2009).

33. I. Bouras et al., arXiv:1008.4072 [hep-ph].

34. B. Betz, J. Noronha, G. Torrieri, M. Gyulassy, I. Mishustin and D. H. Rischke, Phys. Rev. C 79, 034902 (2009).

35. G. Torrieri, B. Betz, J. Noronha and M. Gyulassy, Acta Phys. Polon. B 39, 3281 (2008).

36. L. M. Satarov, H. Stoecker and I. N. Mishustin, Phys. Lett. B 627, 64 (2005).

37. B. Betz, J. Noronha, G. Torrieri, M. Gyulassy and D. H. Rischke, Phys. Rev. Lett. 105, 222301 (2010).

38. D. H. Rischke, Y. Pursun, J. A. Maruhn, H. Stoecker and W. Greiner, Heavy Ion Phys. 1, 309 (1995).

39. A. K. Chaudhuri, Phys. Rev. C 75, 057902 (2007).

40. F. Cooper and G. Frye, Phys. Rev. D 10, 186 (1974).

41. R. B. Neufeld, Eur. Phys. J. C 62, 271 (2009).

42. F. Antinori and E. V. Shuryak, J. Phys. G 31, L19 (2005). 